\title{
Chinesische Arzneimitteltherapie bei Infekten
}

Akute Infekte werden rasch und wirksam behandelt, die Symptome gelindert, der Krankheitsverlauf wesentlich verkürzt - Anhand wichtiger Syndrommuster lassen sich passende Rezepturen schnell und einfach finden josef Hummelsberger

\section{Zusammenfassung}

Für viele Ärzte und Therapeuten ist die chinesische Arzneimitteltherapie wegen der großen Anzahl von Arzneimitteln und Rezepturen eine Hürde. Die Behandlung von Erkältungskrankheiten und Infekten ist jedoch sehr einfach, hervorragend und schnell wirksam. Für Arzt und Patient ist der Erfolg offensichtlich. Und auch bei neuen viralen Infekten wirken die bekannten Rezepturen, da sie nicht erregersondern syndromspezifsch angewendet werden.Dieser Artikel stellt die wichtigen Syndrommuster, die passenden Arzneimittel und Rezepturen praktikabel vor, sodass diese problemlos bei der nächsten Infektwelle eingesetzt werden können.

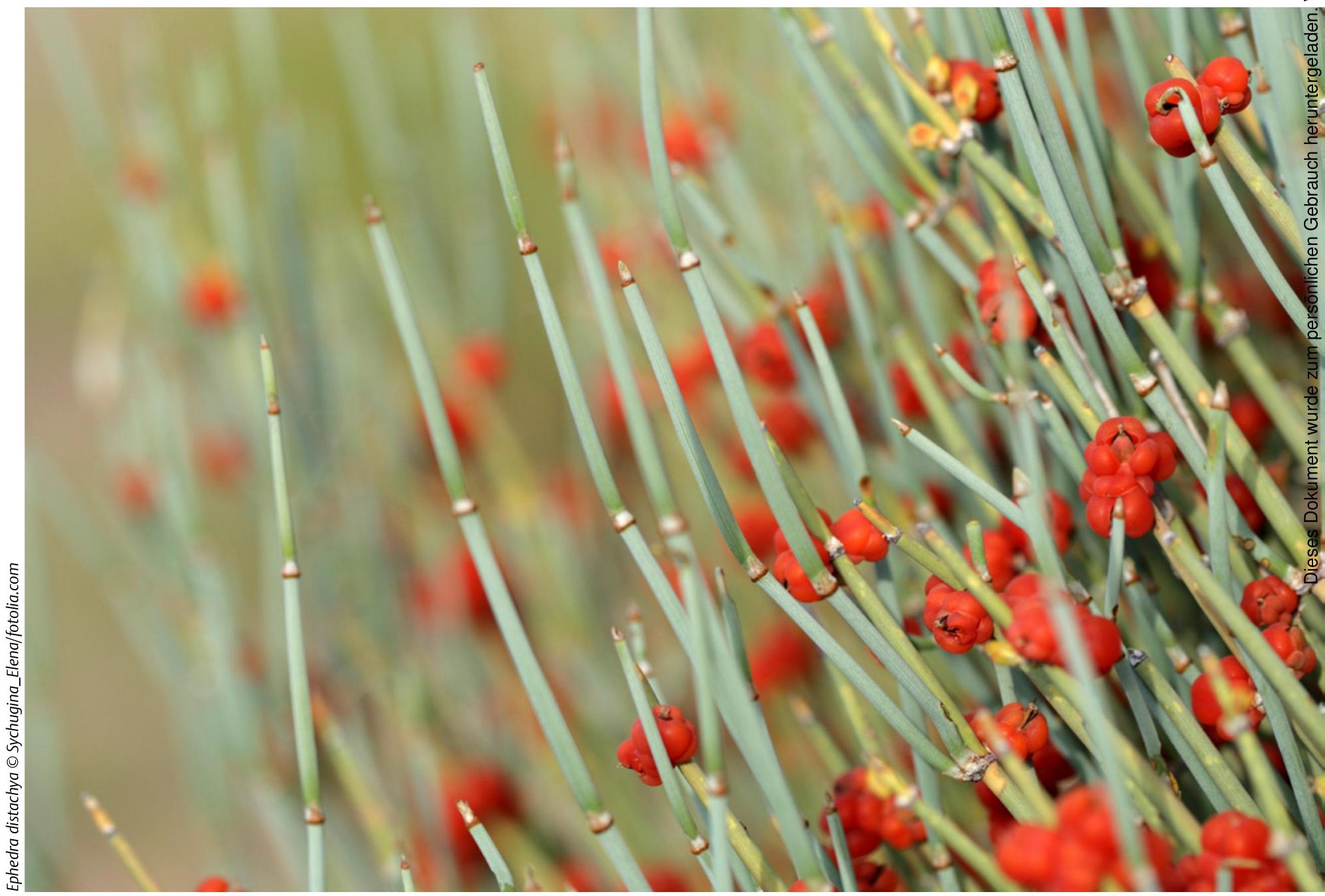




\section{Einleitung}

Die klassische chinesische Medizin (CM) beurteilt die Arzneimitteltherapie als „Herzstück“, als weitaus wichtigste Behandlungsmethode. Akupunktur, Tuina, Diätetik und Qigong (die 4 anderen Säulen der CM) gelten als weniger wirksam bzw. für andere Indikationsgebiete wie Schmerztherapie geeignet. Für viele Ärzte und Therapeuten ist die chinesische Arzneimitteltherapie aber durch die große Anzahl von einzelnen Arzneimitteln und Rezepturen sowie die dann erforderliche sichere Einordnung eines Krankheitsbilds in ein Syndrommuster der CM eine Hürde. Dabei ist die Behandlung von Erkältungskrankheiten und Infekten sehr einfach, die Rezepturen klar, alle Bestandteile sind in Apotheken in Deutschland und den anderen deutschsprachigen Ländern in hoher Qualität erhältlich.

Und für den Arzt besonders wichtig: man hat damit ein neues Werkzeug an der Hand, dass gut und schnell wirksam ist. Dies ist nicht nur durch eine umfassende klinische Erfahrung offensichtlich, Metaanalysen geben positive Wirkhinweise. Die letzten mehr oder weniger bedrohlichen Wellen viraler Mutationen wie SARS, „Vogelgrippe“ und andere wurden in den asiatischen Ländern bevorzugt mit chinesischen Arzneimitteln behandelt. Eine große Reihe von Arzneikräutern wie Lonicera flos (jinyinhua), Forsythiae fructus (lianqiao), Indigo naturalis (Qingdai) weisen antivirale Eigenschaften auf, andere wie Coptis rhizoma (huanglian) wirken durchaus auch antibakteriell. Durch ihre Kombination werden die Effekte oft nicht nur addiert, sondern synergistisch multipliziert

Das Besondere an der CM bei Infekten ist, dass diese Rezepturen - passend eingesetzt - hervorragend wirken, die Symptomatik rasch bekämpfen und für Arzt und Patient der Erfolg offensichtlich ist. Damit ist der Einstieg in die CM leicht, die Behandlung anderer chronischer Krankheitsbilder ist langwierig und eine Erfolgsbeurteilung erfordert mehr Erfahrung.

In diesem Artikel werden die bei uns wichtigen Syndrommuster, die passenden Arzneimittel und Rezepturen praktikabel vorgestellt, sodass diese problemlos bei der nächsten Grippewelle eingesetzt werden können.

\section{Rationale der chinesischen} Arzneimittel

Die traditionelle chinesischen Arzneitherapie (im folgenden TCA) basiert auf:
- Der Biodiversität Chinas (ca. 12\% aller weltweit bekannten Pflanzenarten sind in China beheimatet) und dem daraus resultierenden hohen Anteil arzneilich nutzbarer Arten (ca. 700 offizielle Einträge in der Pharmakopoe der PR China [Pharmacopoeia, 2005 Volume 1, Peoples medical publishing house]).

- Der induktiv-empirischen Systematik der (traditionellen) chinesischen Medizin (Porkert, 1991; Unschuld, 1985) und des - trotz aller Zeitläufe - bewahrten traditionellen Medizinwissens.
(Hempen, 2006, 2007; Bensky, 2004; Scheid, 2009; Porkert 1978, 1984), derzeit erarbeitet die European Medicines Agency (EMEA) ca. 20 europäische Monografien chinesischer Arzneidrogen.

\section{Evidenzlage}

Nach der Evidenzhierarchie der evidenzbasierten Medizin gibt es Cochrane-Reviews mit positiven Evidenzhinweisen zu akuten Infekten der Atemwege (common cold) (Wu T, 2007; Zhang XH et al., 2008) und zu Influenza (Chen, 2010). Bei Letzterem war

\section{Die Orientierung an den Symptomen ermöglicht eine leichte und schnelle Differenzierung der Syndrommuster und des dazu passenden Rezepts.}

- Der Kombination von 2-15 Arzneikräutern zu Rezepturen zur Wirkverstärkung, Nutzung von Synergismen einzelner Arzneipflanzen, Lenkung der Rezepturmischung und Minderung von Geruchs-, Geschmacksbelästigung und von unerwünschten Arzneiwirkungen.

Prinzipiell handelt es sich bei der TCA um eine allopathische Medizin. Zu den Inhaltsstoffen liegen umfangreiche Untersuchungen vor (Chen et al., 2010; Li et al., 2009; Yan, 1999). Wie in der Phytotherapie üblich sind nur sehr selten einzelne Wirkstoffe identifizierbar (Beispiel UDCA, Artemisin), sondern Wirkstoffgemische für die Wirkung verantwortlich. Diese Wirkstoffgemische entfalten nur als solche den gewünschten Effekt (Beispiel Johanniskrautextrakt, Beispiel Behandlung von multiresitenten MRSA durch Qingre-Extrakt (Yu, 2010)). Zur Materia Medica, den Rezepturen und zum System der TCA sind Standardwerke erhältlich das Researchteam aber durch die verschiedenen CM-Diagnosen (s. unten Differenzialtherapie abhängig vom Syndrommuster) und dadurch verschiedenen Rezepturen nicht in der Lage, eine einheitliche Rezeptur zu filtern.

\section{Pathogene Faktoren, Syndrom- muster, Therapiestrategien und Rezepturen}

Aus Sicht der CM werden Infekte durch „äußere pathogene Faktoren“ (6 klimatische Exzesse, liuyin) verursacht: Kälte (algor, han) meist in Kombination mit Wind (ventus, feng), Hitze (calor, re) und Feuer-Toxine (ardor, duhuo), Sommerhitze (aestus, shu) und manchmal auch Feuchtigkeit (humor, shi) sind im Vordergrund. Die Vorstellung der CM ist, dass dieses Pathogen in die Oberfläche (extima, biao) über die Leitbahnen oder Schleimhäute (Funktionskreis Lunge und Großes-Yang-Leitbahnen) eindringen

\section{Bezugsquellen für chinesische Arzneimittel}

Chinesische Arzneimittel sollten generell immer über Apotheken bezogen werden, die Qualität und Reinheit der Drogen gewährleisten. Die TCM-Apotheker-AG ist ein Zusammenschluss engagierter Apotheker, die bundesweit die Versorgung mit hochwertigen Arzneimitteln sicherstellen, die Liste der Apotheken ist unter www.tcm-apo.de abzurufen. In der Schweiz bietet Lian Herbs hohe Qualität (www.lian-chinaherb.ch). In Österreich bietet eine Reihe von Apotheken in Wien, Graz und Innsbruck TCM-Arzneimittel an, die Fa. Plantasia (www.plantasia.at) in Oberndorf ist ein zertifizierter Großhändler, der darüber Auskunft geben kann. 
und zunächst die Wehrenergie (qi defensivum, weiqi) blockieren. Dies führt zu Frösteln, Schüttelfrost , leichtem Fieber, Schwitzen (abhängig vom Pathogen und Stadium), Muskel- und Kopfschmerzen, Müdigkeit und Abgeschlagenheit.

Berücksichtigt wird immer auch

- die allgemeine Konstitution (liegt eine energetische Erschöpfung [depletio/xu] vor?) und

- das Stadium der Erkrankung.

Die hier gebrauchte Differenzierung erfordert lediglich die Orientierung an den Symptomen, zwar sind Grundkenntnisse in der CM nützlich, eine große Erfahrung in der Pulsund Zungendiagnostik sind aber nicht notwendig. Im Folgenden werden die am häufigsten in der Praxis vorkommenden Syndrommuster und ihre Behandlung vorgestellt.

\section{Kälte-Wind-Schädigung in der \\ Oberfläche}

(algor venti, hanfeng)

Typische Symptome sind:

- Exposition des Körpers in einer (zu) kalten

Umgebung, starker Luftzug, Klimaanlage,

- Frieren und Kältegefühl, auch Einwickeln in warme Kleidung oder Decken bringt nur kurzzeitige Erwärmung,

- leichte bis starke Kopfschmerzen, Schmerzen in den Muskeln, Armen, Beinen und / oder im Rücken,

- Naselaufen (sehr viel klares Sekret),

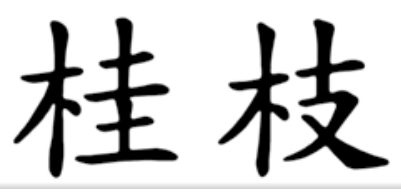

\section{Cinnamomi Cassiae ramuli (guizhi)}

Die Ästchen des Zimtbaums sind warm, süß und scharf. Sie wärmen die Muskeln, speziell auch die Leitbahnen und so den ganzen Körper, Schmerzen und Verkrampfungen werden gemildert. Sie öffnen milde die verschlossenen Poren und induzieren leichtes Schwitzen als Indikator für die Ausleitung des Pathogens. Cinnamomi ramuli (guizhi) wirkt in allen 12 Hauptleitbahnen, besonders am Rücken, Schultern und auch im Unterleib.
- ev. Druck auf der Brust und Husten ohne Auswurf.

- Kein Schwitzen!

- Die Pulse sind nach oben drängend, mächtig und voll, unter Umständen verlangsamt (pp. tardi, repeleti et superficiales).

- Die Zunge ist meist wenig verändert, meist sehr blass und etwas feucht.

In der chinesischen Pharmakopoe stehen eine ganze Reihe von Arzneimitteln bereit, die die Oberfläche wärmen, pathogene Wind-Kälte (algor venti, hanfeng) ausleiten und dadurch auch schmerzstillend und infektbehebend wirken. Alle diese Kräuter sind warm, meist auch von der Geschmackswirkung scharf und aromatisch und wirken auf Oberfläche und den Funktionskreis Lunge (Beispiel Cinnamomi Cassiae ramuli; s. Kasten).

\section{Rezeptur 1 bei Kälte-Wind (algor venti, hanfeng) \\ Ephedra-Dekokt (mahuangtang) \\ - Ephedra herba (mahuang) $9 \mathrm{~g}$ \\ - Cinnamomi ramuli (guizhi) $9 \mathrm{~g}$ \\ - Armeniacae semen (xingren) $6 \mathrm{~g}$ \\ - Glycyrrhizae radix (gancao) $3 \mathrm{~g}$}

Angaben in Tagesdosis für das Dekokt, das bei Kälte-Wind und generell bei Infekten die sinnvollste und wirksamste Form ist. Man nimmt 3-6 Tassen des Dekokts zusammen mit heißem Wasser und sollte dazu Reis essen. Das Ephedra-Dekokt wirkt schnell und sollte zur raschen Erwärmung führen, auch Husten und Druck im Funktionskreis Lunge sollten schnell verschwinden. Ziel ist das Schwitzen; sobald der Patient dies tut, sollte die Einnahme gestoppt werden.

Das Ephedra-Dekokt ist schnell und stark wirkend bei Kälte-Wind (algor benti, fenghan), darf aber nur bei einem solchen Syndrommuster gegeben werden - sonst wird das Schwitzen zu sehr stimuliert, die Säfte geschmälert und es kann auch bei zu hoher Dosis zu Unruhe und Schlafstörungen kommen. Ephedra herba (mahuang) darf nur von Ärzten verschrieben werden, Leistungssportler müssen dies angeben. Denn leider kann daraus Metamphetamin synthetisiert werden, daher steht diese seit über 2500 Jahren verwendete Arznei unter Beobachtung.

Meist kommen die Patienten, die ja noch nicht wissen, wie gut ihnen mit CM geholfen werden kann, nicht im ganz akuten Stadium, sondern wenn der Infekt länger anhält. Auch eine energetische Erschöpfung (depletio/xu) des Qi defensiuvm (Wehrenergie/weiqi) kann zu einem verzögertem Verlauf führen.

Typische Symptome dieses Syndrommusters Kälte-Wind (algor venti/hanfeng) mit verzögertem Verlauf sind

- Frieren und Frösteln, über 3 Tage oder auch 1-2 Wochen,

- mäßiggradige Muskel- und Gliederschmerzen,

- Kopfschmerzen,

- Müdigkeit und Abgeschlagenheit,

- Husten ohne produktiven Auswurf,

- die Nase ist meist verstopft,

- kein Schweiß, oder der Schweiß bringt keine Erleichterung,

- eine blasse Zunge und geschwächte, aber oberflächliche Pulsbilder.

Hier ist die Basis-Rezeptur 2 sehr hilfreich. Sie muss mind. 1 Woche oder länger gegeben werden.

\section{Rezeptur 2 bei Kälte-Wind (algor venti, hanfeng)}

Zimtästchen-Dekokt (guizhitang), modifiziert:

- Cinnamomi ramuli (guizhi) $9 \mathrm{~g}$

- Paeonia lact. radix (baishao) $9 \mathrm{~g}$

- Zingiberis viride rhizoma (shengjiang) $4,5 \mathrm{~g}$

- Jujubae fructus (dazao) $3 \mathrm{~g}$

- Glycyrrhizae radix (gancao) $3 \mathrm{~g}$

Bei Husten kann man dazugeben:

- Armeniacae semen (xingren) $9 \mathrm{~g}$

- Platycodi radix (jiegeng) $6 \mathrm{~g}$

Bei allgemeiner Erschöpfung, leichter Kurzatmigkeit bei Belastung und spontanem Schwitzen kann man kombinieren:

- Astragali radix (huangqi) $9 \mathrm{~g}$

- Atractylodis macro. rhizoma (baizhu) $6 \mathrm{~g}$

- Saposhnikoviae radix (fangfeng) $6 \mathrm{~g}$

(Angaben als Tagesdosen im Dekokt)

Wie generell in der CM kann man eine Vielzahl anderer Arzneimittel mit diesem genialen Rezept aus dem Shanghanlun (ältestes Rezepturbuch der CM, ca. 3. Jh.n.Chr.) zusammen geben. Dieses Rezept ist sicher, wirkt zuverlässig und führt bei richtigem Syndrommuster zur Stabilisierung auch des Qi defensivum (Wehrenergie, weiqi).

\section{Hitze-Wind-Schädigung in der} Oberfläche (calor venti, fengre)

Die wichtige Differenzialdiagnose bei grippalen Infekten ist in der CM immer das Syn- 
drommuster „Hitze-Wind-Schädigung in der Oberfläche“. Bei genauer Befragung und etwas Erfahrung lässt es sich sehr gut vom ersten Bild der Wind-Kälte unterscheiden. Typische Merkmale sind:

- Beginn meist im Frühjahr und Sommer oder auch als Mitbringsel aus wärmeren Regionen,

- meist schneller, manchmal auch schleichender Beginn,

- initial oft Halsschmerzen,

- meist Frösteln, aber weniger ausgeprägt als bei Wind-Kälte,

- initial leichtes Schwitzen,

- Hals, Rachen und auch vorderes Zungendrittel sind gerötet,

- leichte Kopf- und Gliederschmerzen,

- „grippales Syndrom“, Krankheitsgefühl, Müdigkeit, Schwäche und Abgeschlagenheit,

- Lymphknoten und Tonsillen können gerötet sein,

- die Pulse sind leicht beschleunigt und oberflächlich.

Dieses in der Praxis häufige Bild kann sehr erfolgreich mit folgendem Rezept kuriert werden:

\section{Rezeptur bei Wind-Hitze \\ (calor venti, fengre)}

Lonicera-Forsythia-Pulver (yinqiaosan)

- Lonicera flos (jinyinhua) $10 \mathrm{~g}$

- Forsythiae fructus (lianqiao) $6 \mathrm{~g}$

- Platycodi radix (jiegeng) $6 \mathrm{~g}$

- Arctii fructus (niubangzi) $9 \mathrm{~g}$

- Menthae herba (Bohe) $5 \mathrm{~g}$

- Soja semen präp. (Dandouchi) $3 \mathrm{~g}$

- Schizonepetae herba (jingjie) $6 \mathrm{~g}$

\section{Chinesische Arzneimitteltherapie bei Infekten} ist ein Erfolgsmodell: Sie wirkt rasch und erfordert nicht viele Vorkenntnisse des Therapeuten.

- Lophateri herba (danzhuye) $9 \mathrm{~g}$

- Phragmitis rhizoma (Lugen) $9 \mathrm{~g}$

- Glycyrrhizae radix (gancao) 4,5 g

(Tagesdosen im Dekokt)

Diese Rezeptur gibt man in der Regel 3-7 Tage mit der Maßgabe, dass sich der Patient bei einer Verschlechterung wieder vorstellen soll. Bei gegebenem Syndrommuster kann das Lonicera-Forsythia-Pulver (yinqiaosan) neben grippalen Infekten, akuter und chronischer Tonsillitis auch bei Mononukleose verordnet werden.

Für die Langzeiteinnahme sind auch Granulate (gefriergetrocknete Pulverextrakte) oder Fertigpräparate (im Handel als Nahrungsergänzungsmittel) geeignet.

Die Wirkung tritt in der Regel nach 2-3 Tagen ein, die weitere Behandlung richtet sich nach dem Verlauf.

\section{Hitze-Wind (calor venti, fengre)} oder Wind-Kälte (algor venti, hanfeng) dringt in den FK Lunge ein und wird zu Feuer-Glut (ardor, huo) Bei nicht adäquater oder zu später Behandlung kann das Pathogen tiefer in den Funktionskreis Lunge oder sogar den Funktionskreis Magen eindringen und erzeugt hochakute Beschwerden:
- hohes Fieber,

- großen Durst,

- allgemeines Hitzegefühl und starkes Schwitzen,

- u.U. Unruhe des Patienten und Fieberträume,

- Husten mit massivem gelblichem Auswurf,

- schweres Krankheitsgefühl.

Die Zunge ist dann deutlich verändert, sehr rot und meist findet sich ein dicker gelber Belag. Prinzipiell kann dieses Krankheitsbild, dass klinisch-westlich einem schweren bronchopulmonalen Infekt entspricht, mit folgendem Rezept behandelt werden:

\section{Rezeptur bei Hitze im FK Lunge (calor 0 .} pulmonalis, feire)

Modifiziertes Ephedra-Armeniaca-Gypsum-Dekokt (jia maxingshigantang)

- Ephedra herba (mahuang) $6 \mathrm{~g}$

- Gypsum (shigao) $24 \mathrm{~g}$

- Scutellariae radix (huangqin) $9 \mathrm{~g}$

- Houttuyniae herba cum radice (Yuxingcao) $9 \mathrm{~g}$

- Armeniacae semen (xingren) $9 \mathrm{~g}$

- Platycodi radix (jiegeng) $6 \mathrm{~g}$

- Glycyrrhizae radix (gancao) $3 \mathrm{~g}$

(Angaben als Dekokt, g Tagesdosen)

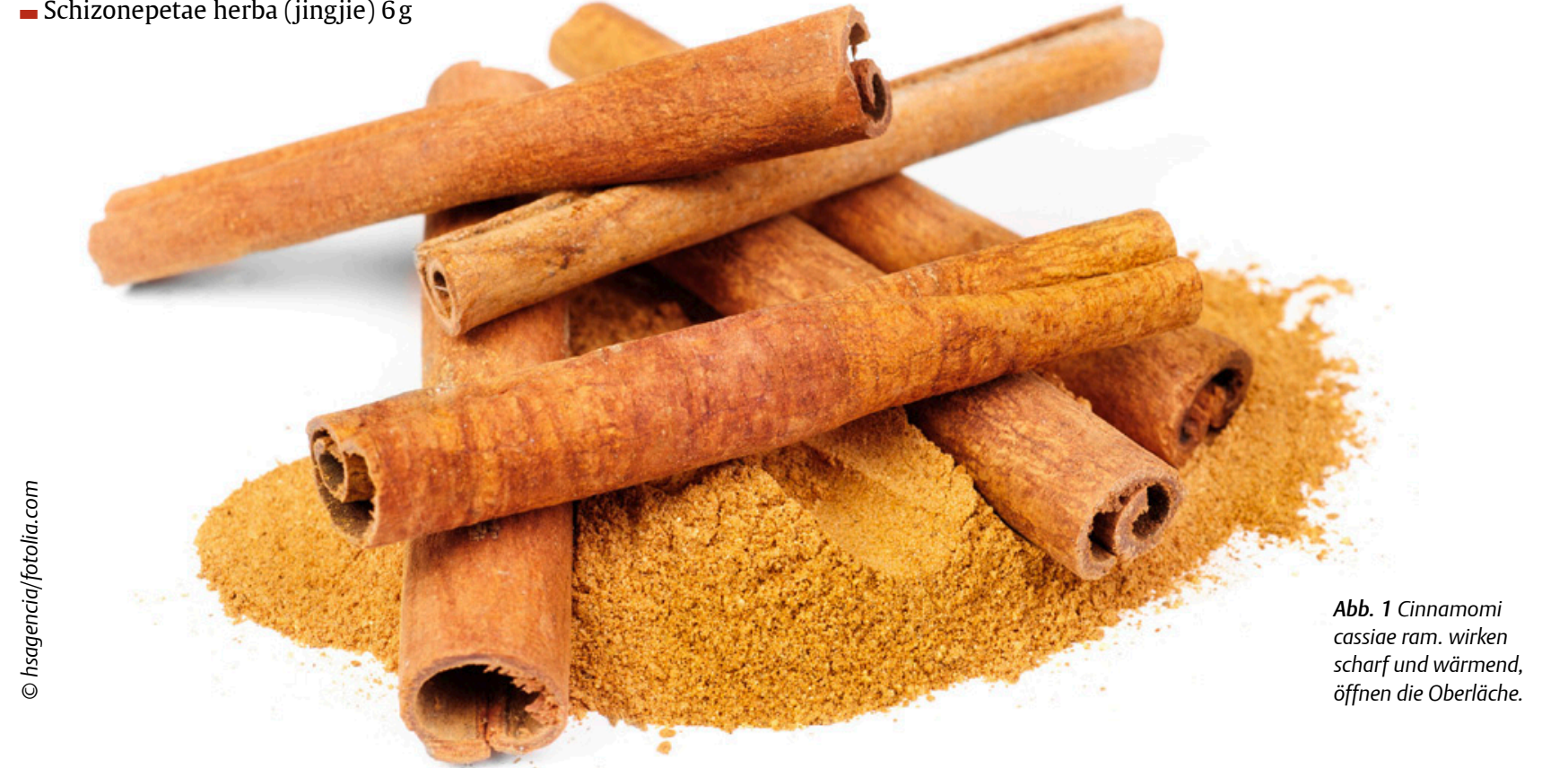




\section{Schulungen in Chinesischer Arzneitherapie}

Diese ärztlichen Fachgesellschaften bieten umfassende Ausbildungen in chinesischer Arzneimittellehre (Dauer meist 2 Jahre):
Bei gegebener Syndromkonstellation führt diese Rezeptur zur raschen Entfieberung, gleichzeitig wird gelber Schleim gelöst und Keuchatmung gestillt. Sie ist eine wirkliche Alternative, besonders wenn bei einem bronchopulmonalen Infekt die Entzün- nehmen. Gleichzeitig besteht aus Sicht der CM eine hohe Gefährdung von Yin, Säften und der Klarheit des shen („Geistes“), also die Gefahr von Kollaps und Bewusstseinsverlust.

Leitsymptome sind:

\footnotetext{
- Societas Medicinae Chinensis (SMS), Ausbildungen regelmäßig in München und Hamburg: www.tcm.edu, ab November 2013 kann die Qualifikation in Chinesischer Arzneitherapie auch im Rahmen eines Masterstudiengangs an der TU München erworben werden: www.tcm.sg.tum.de.

- Deutsche Ärztegesellschaft für Akupunktur (DÄGfA, www.daegfa.de): Ausbildung in Arzneitherapie an 11 Wochenenden und im Rahmen der Qualifikation „Meister der ost-asiatischen Medizin“.

- Über Ausbildungen in der Schweiz kann man sich bei der ASA (www.akupunkturtcm.ch ) und dem Berufsverband der Therapeuten (www.sbo-tcm.ch) erkundigen; am Bodensee und in der Schweiz kann empfohlen werden: www.tcm-advance.de.

- In Österreich bieten eine Reihe ärztlicher Gesellschaften und Universitäten Ausbildungen an (Donau-Universität Krems, Universität Wien und andere), hier gibt es auch eine geregelte Ausbildung für Ärzte in TCA über mindestens 450 Stunden.
}

\section{Aus Sicht der CM entstehen Infekte durch äußere pathogene Faktoren: Kälte, Wind, Hitze, Feuer, Sommerhitze, Feuchtigkeit.}

dungsparameter gegen eine bakterielle Infektion sprechen. Man verordnet diese Rezeptur für 2-3 Tage und bestellt den Patienten dann zur Kontrolle ein.

\section{Sommerhitze (aestus, shure)}

Ein weiteres wichtiges Syndrommuster zur Differenzierung bei akuten Infekten ist im Sommer die sog. „Sommerhitze“ (aestus, shure). Sie verläuft anders und trifft durch die klimatische Konstellation bereits auf einen Patienten mit „offenen Poren" (chin. Couli). So kann sie schneller eindringen und einen akuteren Verlauf

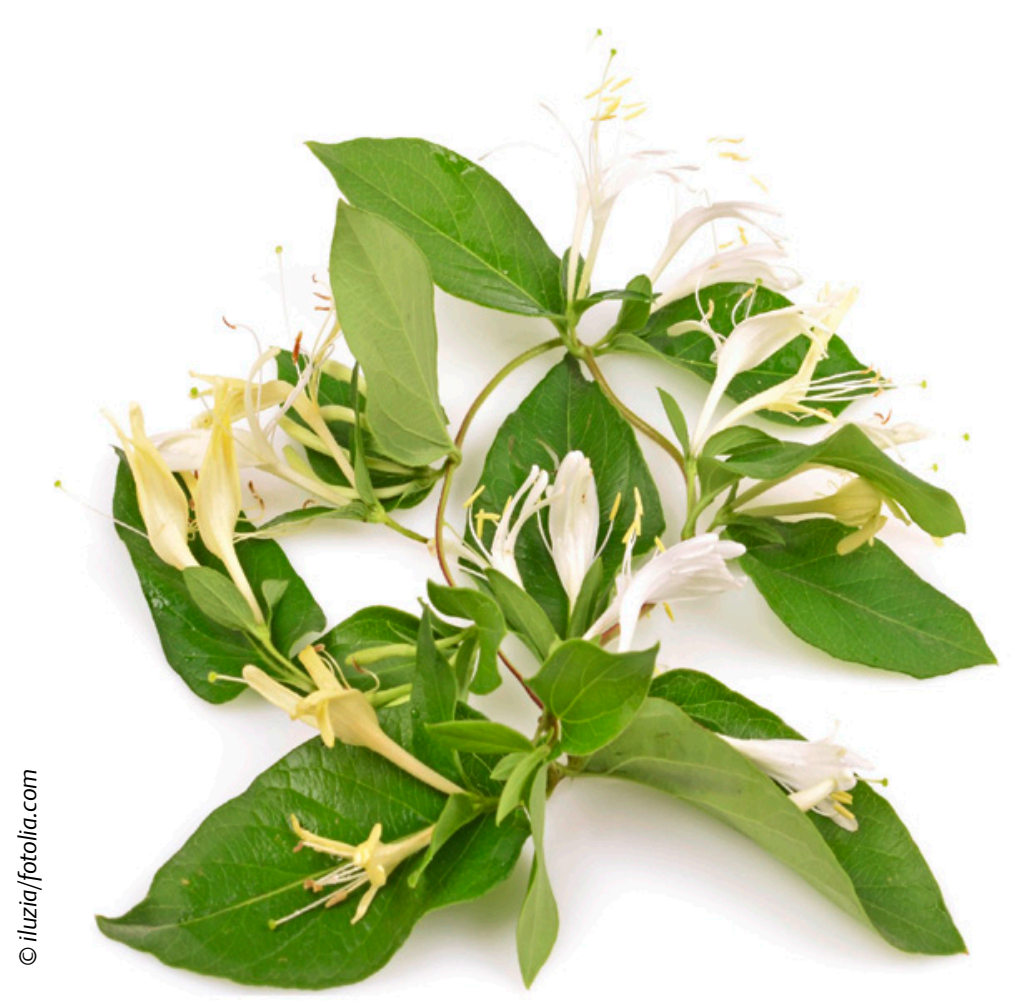

Abb. 2 Japanische Geißblattblüten klären bei Hitze und scheiden Toxine aus.
- akuter Beginn, starkes Krankheitsgefühl, - Frösteln und dennoch Schweißausbrüche, - starke Kopfschmerzen,

- Unruhe bis hin zur Bewusstseinstrübung, - Durchfälle,

- Muskel- und Gliederschmerzen,

- Fieber,

- Durstgefühl.

Die Pulse sind beschleunigt, die Zunge dick belegt.

\section{Basis-Rezeptur Sommerhitze}

Modifziertes Pogostemis-Dekokt zur Wiederherstellung des geraden Qi (jia huoxiangzhengqi tang)

- Pogostemis herba (huoxiang) $9 \mathrm{~g}$

- Perillae foliae et caulis (zisuye) $6 \mathrm{~g}$

- Angelica dahurica radix (baizhi) $6 \mathrm{~g}$

- Moslae herba (ruxiang) $6 \mathrm{~g}$

- Platycodi radix (jiegeng) $6 \mathrm{~g}$

- Atractylodis macro. rhizoma (baizhu) $6 \mathrm{~g}$

- Magnoliae cortex (houpo) $5 \mathrm{~g}$

- Pinelliae rhizoma (banxia) $6 \mathrm{~g}$

- Arecae pericarpium (dafupi) $6 \mathrm{~g}$

- Poria (fuling) $12 \mathrm{~g}$

- Citi retic. pericarpium (chenpi) $6 \mathrm{~g}$

- Glycyrrhizae radix (gancao) $3 \mathrm{~g}$

- Talcum (huashi) $15 \mathrm{~g}$

Dies Dekokt wird für 3, längstens 7 Tage verordnet und führt i.d.R. innerhalb 1-2 Tagen zur deutlichen Besserung, einem schnellen Rückgang besonders der Kopfschmerzen, der Temperaturen und des Durchfalls und zur Stabilisierung des shen („Geist“). Andernfalls muss die Rezeptur dann verändert werden.

\section{Schlussempfehlung}

Die chinesische Arzneimitteltherapie kann bei akuten Infekten in vielen Fällen rasch und wirksam die Symptome lindern und den Krankheitsverlauf wesentlich verkürzen. Eine große Anzahl der einzelnen Arz- 
neidrogen wirken antiviral oder auch antibakteriell, in der Kombination werden diese Effekte synergistisch potenziert, durch die Rezepturen werden Begleitbefunde wie Husten und Verschleimung mitbehandelt.

In der Hand des Arztes ist die chinesische Arzneimitteltherapie eine wichtige Therapiemethode und bietet die Chance, auch bei neuen viralen Infekten wirksame Mittel zur Verfügung zu haben.

Interessenkonflikt: Der Autor erklärt, dass keine wirtschaftlichen oder persönlichen Verbindungen bestehen.

\section{Online zu finden unter}

http://dx.doi.org/10.1055/s-0033-1357225

\section{Literatur}

1 Bensky D. Chinese herbal medicine. Materia medica. Seattle: Eastland Press; 2004, 2008

$\overline{2}$ Brinkhaus B, Hummelsberger L Kohnen R et al. Acupuncture and Chinese herbal medicine in the treatment of patients with seasonal allergic rhinitis: a randomized-controlled clinical trial. Allergy 2004; 59 (9): 953-960

3 Caceres DD, Hancke JL, Burgos RA, Sandberg F, Wikman GK. Use of visual analogue scale measurements (VAS) to assess the effectiveness of standardized Andrographis paniculata extract SHA-10 in reducing the symptoms of common cold. A randomized double blind-placebo study. Phytomedicine 1999; 6: 217-223

$\overline{4}$ Hempen CH, Hrsg. Leitfaden Chinesische Rezepturen. München: Urban und Fischer; 2006

$\overline{5}$ Hempen $\mathrm{CH}$, Fischer T, Wagner H. Leitfaden Chinesische Phytotherapie. 2. Aufl. München: Urban und Fischer; 2006

$\overline{6}$ Lau JT, Leung PC, Wong EL et al. The use of an herbal formula by hospital care workers during the severe acute respiratory syndrome epidemic in Hong Kong to prevent severe acute respiratory syndrome transmission, relieve influenza-related symptoms, and improve quality of life: a prospective cohort study. J Altern Complement Med 2005; 11: 49-55

7 Li XM, Brown L. Efficacy and mechanisms of action of traditional Chinese medicines for treating asthma and allergy. J Allergy Clin Immunol 2009; 123: 297-308

8 Liu C, Douglas RM. Chinese Herbal Medicine in the treatment of acute respiratory tract infections: review of randomized and controlled clinical trials. Clin Infect Dis 1999; 28: 235-236

9 Melchart D, Linde K, Liao JZ, Hager S, Weidenhammer W. Systematic clinical auditing in complementary medicine: rationale, concept, and a pilot study. Altern Ther Health Med 1997; 3: 33-39

$\overline{10}$ Porkert M. Die theoretischen Grundlagen der chinesischen Medizin. 3. Aufl. Basel: AMS; 1991 $\overline{11}$ Porkert M. Klinische chinesische Pharmakologie. Heidelberg: Fischer; 1978

$\overline{12}$ Porkert M. Neues Lehrbuch der chinesischen Diagnostik. Dinkelscherben: Phainon; 1993

$\overline{13}$ Scheid V et al. Chinese herbal medicine. Formulas and strategies. Seattle: Eastland Press; 2009

$\overline{14}$ Zhenxiang Z. Zum Gebrauch von chinesischen Arzneimitteln bei Erkältungskrankheiten. Chin Med 2002; 2002; 17 (4): 127

\section{Anleitungen für Dekokte, Granulate}

Sie können von der Homepage des Verfassers die Anleitungen zur Einnahme und Herstellung der Dekokte herunterladen: www.hummelsberger. net/downloads.htm

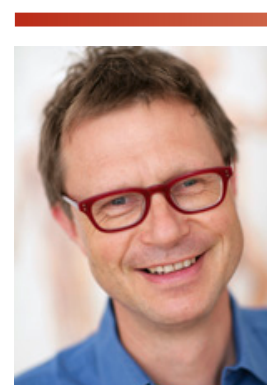

Dr. Josef Hummelsberger

Vize-Präsident der SMS (Societas medicinae sinensis)

Hackenstr. 7

80331 München

praxis@hummelsberger.net

www.hummelsberger.net

Studium der Medizin in Göttingen und München, Facharztausbildung zum Internisten. Seit 1982 Ausbildung in Akupunktur bei verschiedenen Fachgesellschaften. Seit 2005 in eigener Praxis mit dem Schwerpunkt Akupunktur \& Chinesische Medizin tätig. Seit 1997 Dozent und Gastdozent für Akupunktur und Chinesische Medizin bei ärztlichen Fachgesellschaften, Akademien und Universitäten (SMS, DGfAN, DÄGfA, EIOM, Ärztekammer Westfalen-Lippe, Donau-Universität Krems). 